Ebisu Ebisu

Études japonaises Études japonaises

47 | printemps-été 2012

Catastrophes du 11 mars 2011, désastre de

Fukushima : fractures et émergences

\title{
Séisme et tsunami du 11 mars 2011 : spatialisation de la catastrophe
}

The Spatial Dimension of the 11 March Tsunami Disaster

地域的特色からみた津波効策の盲点

\section{Rémi Scoccimarro}

\section{OpenEdition}

Journals

Édition électronique

URL : http://journals.openedition.org/ebisu/204

DOI : 10.4000/ebisu.204

ISSN : 2189-1893

Éditeur :

Institut français de recherche sur le Japon (UMIFRE 19 MAEE-CNRS), Maison franco-japonaise

Édition imprimée

Date de publication : 1 juin 2012

Pagination : 13-25

ISSN : 1340-3656

Référence électronique

Rémi Scoccimarro, "Séisme et tsunami du 11 mars 2011 : spatialisation de la catastrophe », Ebisu [En ligne], 47 | printemps-été 2012, mis en ligne le 03 avril 2014, consulté le 02 mai 2019. URL : http:// journals.openedition.org/ebisu/204; DOI : 10.4000/ebisu.204 


\title{
Séisme et tsunami du 11 mars 2011 Spatialisation de la catastrophe
}

\author{
Rémi SCOCCIMARRO
}

Spatialiser la catastrophe, révéler sa dimension géographique permet de mieux la comprendre et d'en saisir les enjeux. Ainsi, à la différence du séisme de Kobe en 1995 (dit Hanshin Awaji daishinsai 阪神・淡路大震災), la localisation du séisme et du tsunami du 11 mars 2011 dans le Sanriku 三陸 est peu surprenante. En revanche, la destruction de villages parmi les mieux protégés au monde nous interpelle. Elle pose en particulier la question des limites d'un certain modèle de développement urbain et des choix faits au $\mathrm{Xx}^{\mathrm{e}}$ siècle pour la protection des populations.

En nous situant d'abord à l'échelle régionale, puis à l'échelle locale, nous montrerons que malgré la diversité des contextes et des conjonctures, la nature catastrophique du 11 mars est aussi le résultat de logiques bien identifiables d'aménagement du territoire.

\section{Quel Japon détruit ?}

En premier lieu, le séisme du 11 mars 2011 malgré sa durée, près de dix minutes en comptant les répliques, a été relativement peu destructeur. Son mouvement, essentiellement latéral, a limité l'impact, alors que lors de

Géographe, maître de conférences en langue et civilisation japonaises à l'université de Toulouse-Le Mirail. Ses recherches portent sur l'aménagement des fronts de mer et sur les recompositions urbaines et démographiques du Japon contemporain, et depuis le 11 mars 2011 sur la catastrophe du Japon de l'Est. 
vibrations verticales les constructions parasismiques sont moins opérantes (Shimamura 2011).

La ville millionnaire de Sendai 仙台, métropole la plus proche de l'épicentre, a ainsi subi peu de dégâts liés directement au séisme. Au sud, dans la baie de Tokyo, les terrains gagnés sur la mer, traditionnellement plus vulnérables, ont été affectés par des phénomènes de liquéfactions (ekijōka 液状 化) spectaculaires, mais qui n'ont pas pour autant été meurtriers (Komachi, entretien 2011). Par exemple, dans le quartier de Shin.urayasu 新浦安 (département de Chiba 千葉) construit sur remblais dans les années 1980, la plupart des bâtiments sont restés habitables. C'est un enseignement rassurant, qui montre que les villes, en particulier les logements issus du boom des manshon マンション ${ }^{1}$ des années 1998-2005, ont été effectivement construits aux normes ${ }^{2}$.

Dans les vastes zones industrialo-portuaires, la secousse n'a provoqué qu'un seul accident majeur, une explosion de gaz à Ichihara 市原 (baie de Tokyo, département de Chiba), et des incendies dans les réservoirs d'hydrocarbures comme dans les ports de Sendai et de Kesennuma 気仙沼. Ils furent également spectaculaires mais relativement marginaux au regard des concentrations industrielles.

En revanche, le littoral du Nord-Est a été affecté par une subsidence de l'ordre de $0,8 \mathrm{~m}$ à $1 \mathrm{~m}$ en moyenne ${ }^{3}$. Cela a abaissé le niveau de protection des brise-lames et des digues, rendant aujourd'hui la plupart des ports inutilisable ${ }^{4}$.

1. Habitat collectif en hauteur.

2. Ce qui n'allait pas de soi, comme l'a montré l'affaire de l'architecte Aneha Hidetsugu 姉歯秀次 dont on apprit en 2005 qu'il avait falsifié les données relatives à la résistance parasismique d'au moins 98 immeubles d'habitation pour économiser sur le volume d'acier (Asahi shinbun du 24 novembre 2005).

3. Entretiens auprès des responsables locaux des villes d'Ōfunato 大船渡, Kesennuma et Ishinomaki 石巻 les 9 et 11 août 2011, entretien du 8 juillet 2011 avec Miyake Satoshi 三宅諭 (architecte, maître de conférences au département d'agriculture de l'université d'Iwate à Morioka, chargé d'étude pour la reconstruction de la commune de Yamada à Iwate) et relevés personnels de terrain en juillet et août 2011 (voir carte ci-après).

4. En particulier pour les chalutiers de plus de $30 \mathrm{~m}$ destinés à la pêche au large comme dans le port de Kesennuma (département de Miyagi) ou pour les baleiniers du port d'Ayukawa 鮎川 (commune d'Ishinomaki, département de Miyagi). 


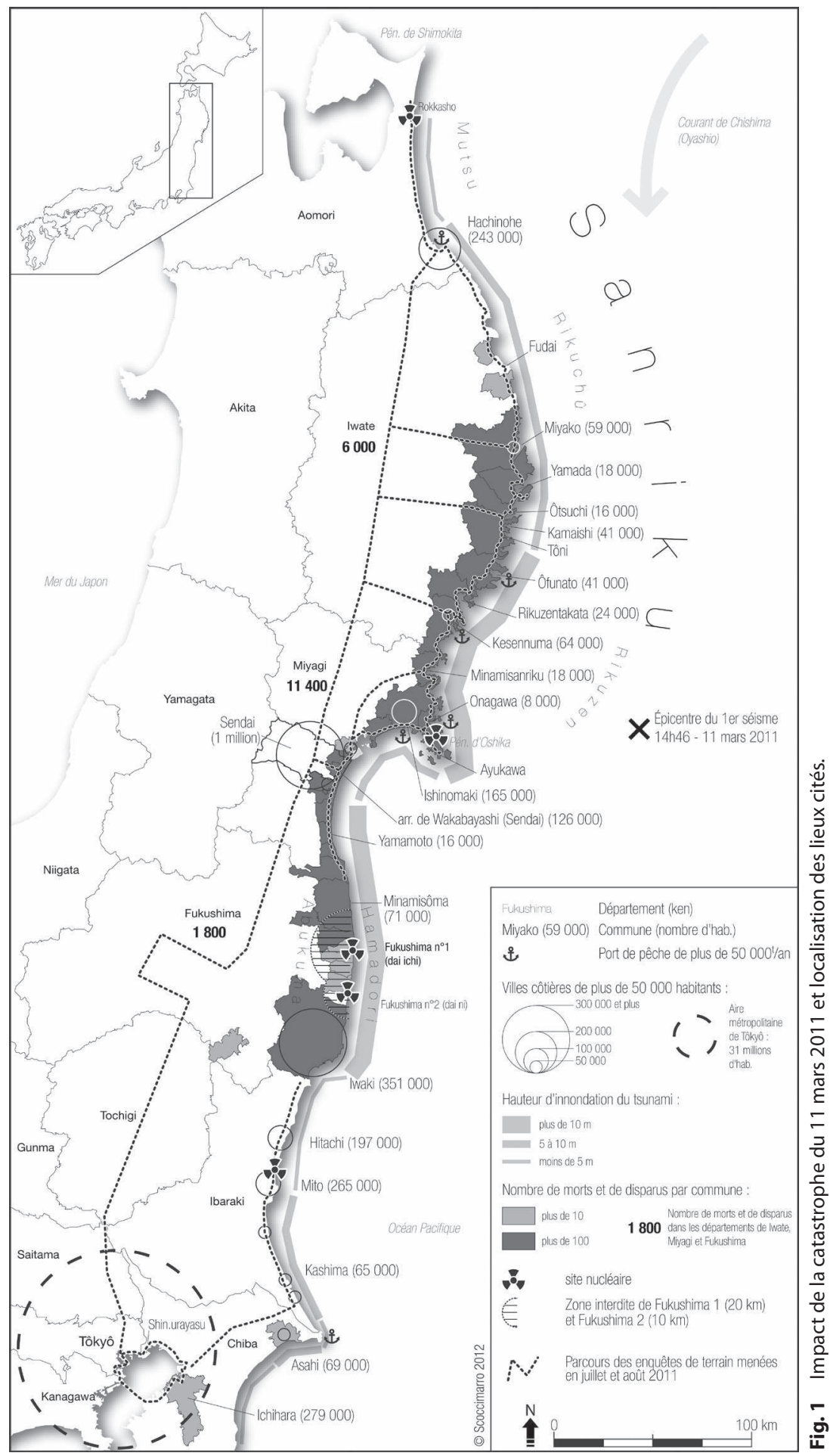


Dans certaines villes ${ }^{5}$, les coupures d'électricité et du réseau de téléphonie cellulaire dues au séisme ont désactivé les systèmes d'alerte anti-tsunami. Cela explique que de nombreuses personnes, immobilisées physiquement par les secousses pendant une dizaine de minutes, ont tardé à fuir pendant la vingtaine de minutes qui a précédé le deuxième choc, celui du tsunami.

L'impact de celui-ci a donc été fonction d'invariants, comme la nature des sols ou la topographie des côtes, mais aussi de l'état de préparation des populations et de leur rapport géo-historiques à la mer et aux tsunamis. Dans cette optique, il faut distinguer la situation du Sanriku, au nord, et celle, très différente, des plaines côtières du sud du département de Miyagi 宮城 et de celui de Fukushima 福島.

\section{«Tsunami Sanriku»}

Le littoral du Sanriku s'étend de la péninsule d'Oshika 牡鹿 (Miyagi), au nord-est de Sendai, à celle de Shimokita下北 (Aomori 青森), à l'extrémité nord de Honshū 本州 (voir fig. 1). C'est une des régions du Japon les plus exposées aux tsunamis, en avant-poste des zones de subduction du Pacifique qui les génèrent. Sa topographie de ria (vallée côtière étroite) la rend plus vulnérable : à l'approche des côtes, les flots gagnent mécaniquement en hauteur, au fur et à mesure du resserrement de la vallée, provoquant des vagues déferlantes de 40 mètres de hauteur ${ }^{6}$. Les villes et les bourgs côtiers de cette région sont ainsi régulièrement frappés par les tsunamis, dont les plus violents ont été ceux de 1896 (tsunami de Meiji Sanriku 明治三陸津波, 22000 morts), de 1933 (tsunami de Shōwa Sanriku 昭和 三陸津波, 3000 morts) et de 1960 (tsunami du Chili, 142 morts).

Les populations du Sanriku sont donc coutumières de ce type d'événements et elles ont développé des comportements adaptés : sur terre, il s'agit de « la fuite à tout va ", tenden-ko てんでんこ en patois d'Iwate 岩手. Le littoral rocheux est alors un avantage, car on atteint vite des hauteurs suffisantes.

5. Attesté à Kesennuma (Satō, entretien 2011) et Ishinomaki (Saitō, entretien 2011), mais rapporté aussi pour d'autres communes.

6. Mais la hauteur des vagues déferlantes ne correspond pas à la hauteur de l'inondation des masses d'eau déplacées par le tsunami qui atteint rarement plus de 16 m (Kawata 2010). 
Pour les pêcheurs, c'est okidashi 沖出し : prendre le large où les vagues sont moins hautes, pour préserver les bateaux et éviter qu'ils ne servent de projectiles sur la côte. On sait également lire les signes de l'arrivée imminente du tsunami lors d'un retrait brutal des eaux sur les plages et dans les ports.

Enfin, depuis l'après-guerre, les villes, les bourgs, les villages et même les hameaux côtiers du Sanriku sont enserrés dans des digues de béton, en particulier dans le département d'Iwate où elles atteignent souvent plus de $10 \mathrm{~m}$ de hauteur?

Les tsunamis n'ont cependant pas restreint le peuplement du littoral du Sanriku. Cette partie du Tōhoku 東北 est historiquement une région pauvre. Elle ne bénéficie pas d'un enneigement semblable à l'ouest du Tōhoku (Niigata 新潟, Akita 秋田), qui est favorable à la riziculture. Les récoltes sont, de surcroît, à la merci du yamase 山背, un vent froid d'été qui stoppe la croissance du riz et peut diviser par trois les rendements agricoles des années où il a soufflé (Yamamura 2008).

La partie littorale est restée isolée de l'axe d'urbanisation Sendai-Morioka 盛岡, malgré quelques liaisons ferroviaires comme la ligne côtière mise en service dans les années $1980^{9}$.

En revanche, le Sanriku baigne dans les eaux les plus poissonneuses au monde. Elles le doivent au courant froid de Chishima 千島海流 (Oyashio 親潮), alors que du sud remonte le puissant courant tropical Kuroshio 黒潮. Le mélange des eaux est à l'origine d'une richesse halieutique exceptionnelle. Elle explique l'implantation d'une série de petits bourgs portuaires de dix à vingt mille habitants, dans des havres naturels, encadrés par de petites villes de l'ordre de 50000 habitants comme Kesennuma, Ōfunato, Miyako 宮古 ou Kamaishi 釜石. Alors que la pêche hauturière a diminué, et même si les prises ont plutôt une faible valeur marchande, l'abondance de

7. Source : Kaigan bōchō teibō setchi ichiran 海岸防潮堤防設置一覧 (Tableau des hauteurs et longueurs de digue pour les côtes du département d'Iwate), document interne fourni par le Bureau de l'aménagement et de la planification du département d'Iwate le 8 août 2011, daté du 31 mars 2009 .

8. Appelé aussi, localement, kigakaze 飢餓風 (vent de la faim), vent du nord généré par les masses d'air polaire stationnées sur la mer d'Okhotsk et qui longe les reliefs de la bande côtière est du Tōhoku.

9. La ligne des rias (リアス線 riasusen), à une seule voie, entièrement détruite par le tsunami. 
la ressource permet le maintien du secteur de la pêche et de l'aquaculture, avec une moyenne d'âge des pêcheurs de dix ans inférieure à celle nationale (Kataishi, entretien 2011).

Le tourisme, basé sur la gastronomie des produits marins (Saint-Jacques, oursins, huîtres) et sur la beauté des paysages de falaises, offre un autre débouché. Mais cette activité est limitée par l'enclavement de cette région éloignée de la mégalopole et insuffisamment raccordée aux voies de communication principales.

\section{Les plaines côtières de Sendai et de Hamadōri}

Le sud du littoral frappé par le tsunami, d'Ishinomaki à Kashima 鹿嶋 (département d'Ibaraki 茨城), offre un profil très différent. Il s'agit pour l'essentiel de côtes basses, de plaines alluviales inondables mais théoriquement moins vulnérables aux tsunamis. Elles sont en effet formées d'un cordon littoral régulier où, à l'inverse des rias du Sanriku, les vagues d'un tsunami perdent en vigueur et en hauteur au fur et à mesure qu'elles avancent dans les terres.

Dans la périphérie de Sendai, ce littoral a été urbanisé et industrialisé, souvent gagné sur la mer comme à Ishinomaki. Il est protégé par des digues qui dépassent rarement huit mètres de hauteur. Celles-ci sont par ailleurs souvent d'anciennes levées de terre, simplement recouvertes de galets puis de béton, et qui n'ont pas résisté à la submersion du 11 mars 2011.

Plus au sud, dans le département de Fukushima, ce sont de petites plaines côtières rizicoles, au pied des plateaux de l'Abukuma 阿武隈, sur le littoral du Hamadōri 浜通り. Depuis la haute croissance économique (1955-1973), ces bourgs ruraux hébergent des centrales électriques thermiques et nuclé$a_{i r e s}{ }^{10}$. Cette dernière énergie est proposée au département de Fukushima par l'opérateur Tepco dès 1955 quand le gouvernement japonais lance un programme nucléaire civil. C'est alors un moyen pour la région de pallier la position excentrée du littoral par rapport à l'axe de développement le long de la plaine de Fukushima, dans le prolongement de la mégalopole ${ }^{11}$. La présence des centrales permet d'enrayer le déclin démographique, comme

10. Pour alimenter la mégapole de Tokyo.

11. Dans l'axe Tokyo-Utsunomiya 宇都宮-Kōriyama 郡山-Fukushima-Sendai. 
dans le bourg d'Ōkuma 大熊 où se trouve la centrale de Fukushima Daiichi. Mais sa population ne gagne que 2000 habitants entre 1966 (8 000) et 2011 (10 000), montrant les limites de ce type d'implantation en termes d'aménagement du territoire.

\section{La fabrique de la catastrophe}

Un examen à grande échelle géographique ${ }^{12}$ permet d'analyser la catastrophe du 11 mars comme étant aussi le résultat de choix et de modèles de développement qui ont contribué à faire du tsunami une catastrophe majeure. Ainsi, la conjonction entre l'implantation de quartiers d'habitation denses, avec des populations peu au fait des dangers de la mer, sur des zones basses vulnérables, a été un facteur important de destruction et de mortalité. Tout comme la foi dans les digues géantes qui a donné un dangereux sentiment de sécurité.

\section{Extension urbaine et dichotomie socio-spatiale dans la métropole de Sendai}

Sendai n'est pas une ville côtière à l'origine, mais une ville " au pied du château " (jökamachi 城下町) fondée au début du XVII ${ }^{\mathrm{e}}$ siècle, à partir d'une forteresse bâtie sur des hauteurs, à $12 \mathrm{~km}$ du front de mer actuel. La ville marchande se développe en contrebas, alors que la plaine alluviale vers la mer est aménagée pour la riziculture inondée. C'est une zone basse, humide, dont les marais sont peu à peu remblayés et les rivières endiguées. Des levées de terre sont construites le long de ces dernières et sur le littoral, alors que les habitations rurales sont surélevées sur des buttes, naturelles ou artificielles, pour faire face aux inondations.

À partir du xx siècle, avec la construction de ports modernes, l'urbanisation progresse vers la mer. Lors de la haute croissance économique, le littoral d'Ishinomaki à Shiogama 塩釜 (Sendai) se transforme en zone industrialoportuaire, tandis que la banlieue de Sendai se développe en direction de la

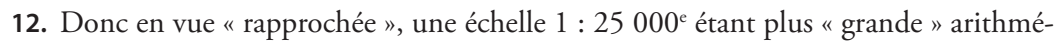
tiquement qu'une échelle $1: 100000^{\mathrm{e}}$. 
mer. C'est particulièrement le cas de l'arrondissement de Wakabayashi 若林 qui est intégré à la commune de Sendai en 1989. Aujourd'hui encore, seule sa partie ouest est classée en zone urbaine ${ }^{13}$, alors que l'est, séparé par une voie express, est encore majoritairement agricole. Les rizières y dominent mais beaucoup ont été transformées en champs secs et forment le sud-est de la ceinture maraîchère de Sendai.

Depuis les hameaux ruraux d'origine, un mitage urbain sous la forme de lotissements résidentiels s'est développé, remplaçant les terres agricoles, à proximité des rivières ou du front de mer. À l'abri de digues, mais sur des terrains très bas de $4 \mathrm{~m}$ à $2 \mathrm{~m}$ d'altitude, et inondables si ces premières cèdent. La présence de la mer est presque imperceptible et beaucoup des habitants témoignent aujourd'hui du fait qu'ils n'avaient jamais imaginé l'éventualité d'être victime d'un tsunami ${ }^{14}$.

Les dunes littorales boisées de conifères formaient un rideau que les premières vagues du tsunami ont ouvert, alors que les zones de refuges possibles sont très éloignées. Beaucoup d'habitants ont ainsi été surpris et n'ont commencé à fuir que lorsqu'ils ont aperçu et entendu les vagues, c'est-à-dire trop tard.

Cela révèle la dimension éminemment sociale de la catastrophe, dans le prolongement de la dichotomie socio-spatiale héritée des jōkamachi basée sur la vulnérabilité. Ces quartiers urbanisés dans les années 1970 et 1980 hébergent les classes urbaines populaires de la métropole. Ainsi, l'arrondissement de Wakabayashi est celui dont les terrains, à usage résidentiel comme agricole, sont les moins chers de la ville ${ }^{15}$.

13. Soit en densely inhabited discrict (ou jinkō shüchū chiku 人口集中地区), une des définitions de l'espace urbain au Japon, secteur de plus de 5000 habitants et de densité supérieure à $4000 \mathrm{hab} / \mathrm{km}^{2}$.

14. D'après le témoignage d'une habitante de l'arrondissement de Wakabayashi, recueilli le 7 juillet 2011 à Sendai et dont la maison, à $4 \mathrm{~km}$ de la côte dans le quartier de Rokugō 六郷, a été partiellement détruite.

15. Source : annuaire statistique de la ville de Sendai, 2010. 


\section{L'exosquelette des infrastructures du Sanriku}

Au contraire de la plaine de Sendai, on pourrait penser que les populations du Sanriku ont mieux réagi et que la mortalité y a été plus faible. Il n'en est rien. Là, c'est le choix d'urbaniser des zones dangereuses par la construction de digues géantes ${ }^{16}$ qui a affaibli les réflexes de survie de ces habitants sur le long terme. Ces digues géantes ont diffusé un sentiment de protection.

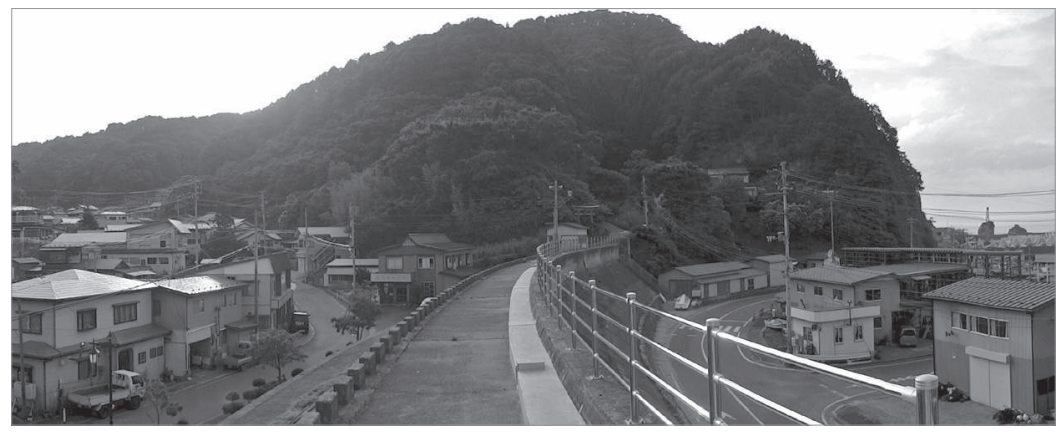

Fig. 2 Le village de Fudai (Iwate) dont les habitations sont protégées par une digue de $15 \mathrm{~m}$, le 9 juillet 2011.

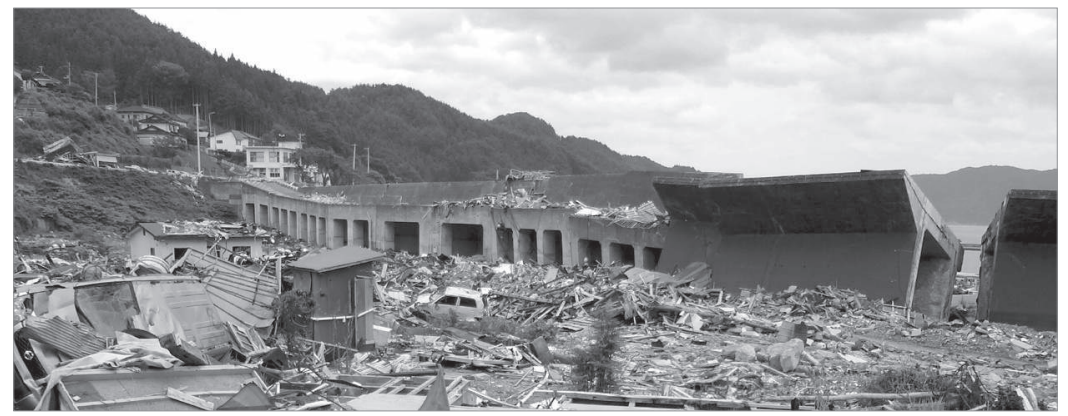

Fig. 3 Tōni, commune de Kamaishi (Iwate), le 8 juillet 2011.

16. Des choix aussi politiques, dans une logique électorale et le souci de soutenir l'économie locale. 
Malgré leur taille et leur présence systématique, les digues du Sanriku ont été inopérantes. Certes l'événement fut exceptionnel, mais pas tant si on le compare au tsunami de 1896, dont les vagues déferlantes atteignirent également près de $38 \mathrm{~m}$. Aussi, il est surprenant de constater que le périmètre d'inondation et de destruction du tsunami du 11 mars suit quasiment le même contour que celui des tsunamis de référence ${ }^{17}$. Les digues d'Iwate furent pourtant des modèles et des références mondiales dans l'art de la protection anti-tsunami.

La plus haute, celle de Fudai-mura 普代村 (Iwate) avec 15 m, est une des seules à être intactes aujourd'hui (fig. 2). Elle a contenu les eaux du tsunami, parce que l'inondation, à ce point de la côte abrité par un cap, fut relativement faible avec moins de $10 \mathrm{~m}$. D'autres digues, de conception plus récente, ont été éventrées et submergées, comme celle de Tōni 唐丹 (fig. 3) à Kamaishi (Iwate).

Renversées, défoncées, ou simplement submergées, on peut émettre l'hypothèse que ces digues ont paradoxalement accru la vulnérabilité de ces bourgs en permettant la conquête de zones basses à proximité des embouchures des rivières, transformées en rizières puis en zones habitées comme à Minamisanriku 南三陸 ou Rikuzentakata 陸前高田 ${ }^{18}$.

À l'abri des digues géantes, les pêcheurs ont pu s'installer au plus près de la mer. Cette situation découlait souvent de la demande même des habitants, comme par exemple à Tarō 田老 (Iwate) où lors de la reconstruction qui a suivi le tsunami de 1933, le déplacement des villages sur les hauteurs un temps envisagé fut abandonné. La même question se pose aujourd'hui et butte sur la même volonté des pêcheurs de revenir exactement sur le lieu de leurs anciennes habitations (Itō et al. 2011 et enquête de terrain de juillet 2011).

17. De 1896 et de 1933 (Iwate-ken 2004), comparé aux relevés de terrain lors des missions sur la totalité de la côte frappée par le tsunami à Miyagi, à Iwate et à Aomori (missions du 4 juillet au 10 juillet 2011 et du 7 au 11 août 2011), en confirmation des premiers relevés sur carte au $25000^{\mathrm{e}}$ présentés par la Société japonaise de géographie (Nihon chiri gakkai 日本地理学会) et établis à partir des photos aériennes des 13, 14 et 15 mars 2011 fournies par l'Institut géographique du Japon (Kokudo chiri in 国土地理 院) sur l'ensemble de la zone côtière de Chiba à Ibaraki.

18. Deux villes aujourd'hui entièrement détruites. 


\section{Conclusion}

Tenter de saisir la catastrophe du 11 mars par ses différentes échelles (régionale, locale) permet, entre autres, de comprendre sa nature et certains des enjeux qu'elle soulève en matière d'aménagement du territoire et de protection des populations.

Dans le Sanriku, comme dans la plaine de Sendai ou le long du littoral de Fukushima, la confiance en la protection " dure " a primé sur la protection "molle", celle des réflexes ancestraux et du maintien de zones non-constructibles, finalement plus efficaces que les masses de béton. Le tsunami du 11 mars a montré que, tel un exosquelette, une fois la coquille des digues percée, les bourgs et leurs populations moins conscientes du risque se sont retrouvés particulièrement exposés.

Cela incite aujourd'hui à envisager la révision du principe des solutions purement techniques, pour protéger les populations (Itō et al. 2011). Il faudra reconstruire des digues, certainement, mais en gardant bien à l'esprit qu'elles ne protègent pas de tous les tsunamis.

Il faut aussi revoir la conception des villes et des bourgs du Sanriku, en particulier à l'intérieur des périmètres inondables qui se sont, eux, révélés très fiables. D'autant que les réflexes traditionnels comme le tendenko ne peuvent être la seule solution, en particulier dans des régions vieillissantes. Le 11 mars 2011, la majorité des décès dans le Sanriku concerne des personnes âgées de plus de 65 ans $^{19}$, moins mobiles, qui ne peuvent pas fuir seules ou aussi vite que les autres.

La reconstruction des côtes dévastées ouvre désormais la question des choix à faire : des projets dans les cartons pour des digues à $15 \mathrm{~m}$ chez les ingénieurs, à la volonté politique de créer des périmètres non constructibles dans les zones inondables, au prix de la résistance de populations qui expriment leur intention de revenir sur leur lieu d'habitation.

12 mars 2012

19. Ainsi, sur les 435 morts et disparus de la commune d'Ōfunato (Iwate), plus de $57 \%$ (249) étaient âgés de 71 à 100 ans, seulement $11 \%$ (51) étaient âgés de 21 à 50 ans (source : document interne de la mairie d'Ōfunato, daté du 2 août 2011 et fourni le 9 août 2011). 


\section{Sources}

\section{DAUPHINÉ André, 2001}

Risques et catastrophes, observer, spatialiser, comprendre, gérer, Paris, Armand Colin.

\section{HIRAOKA Akitoshi 平岡昭利, 2006} [2 éd.]

Tōhoku. Chizu de yomu hyakunen 東北、地図で読む百年 (Le Tōhoku. Un siècle vu à travers les cartes), Tokyo, Kokin shoin 古今書院.

ITŌ Shigeru 伊藤滋, OKUNO Masahiro 奥野正寛, ŌNISHI Takashi 大西隆 et HANAZAKI Masaharu 花崎正晴 (dirs.), 2011

Higashi Nihon daishinsai fukkō e no teigen 東日本大震災復興への提言 (Propositions pour la reconstruction après le grand séisme de l'Est du Japon), Tokyo, Tokyō daigaku shuppankai 東京大学出版会.

IWAFUNE Masaki 岩船昌起, 2011 «Tsunami saizensen de no seizon kankyō o kangaeru : Miyako shiyakusho kara no "tsunami eizō" no kaiseki » 津波最前線での生存環境を考える——宮古 市役所からの「津波映像」の解析 (Réflexion sur les conditions de survie en première ligne de front du tsunami : analyse à partir des images du tsunami produites par la mairie de Miyako), Chiri, 56 (7) : 20-29.

\section{Iwate-ken 岩手県 (département} d'Iwate), 2004 Iwate-ken jishin/tsunami shimyurēshon oyobi saigai sōtei chōsa ni kansuru hōkokusho 岩手県地震・津波シミュレー ション及び災害想定調査に関する報告書 (Simulation des séismes et des tsunamis pour le département d'Iwate et enquête sur les hypothèses de catastrophes naturelles), département d'Iwate.

KAWATA Yoshiaki 川田嘉昭, 2010 Tsunami saigai, gensai shakai o kizuku 津波災害、減災社会を築く (Désastres par tsunamis, bâtir une société qui en limite l'ampleur), Tokyo, Iwanami shoten 岩波書店.

Nihon chizu sentā 日本地図センター Chizu de miru Sendai no hensen 地図で見る仙台の変遷 (Les mutations de Sendai vues par les cartes), [série de cartes topographiques au $25000^{\mathrm{e}}$ de l'agglomération de Sendai des années 1905, 1928, 1946, 1964, 1977 et 1998, et fascicule explicatif de 26 p.], Tokyo, Nihon chizu sentā.

SHIMAMURA Hideki 島村英紀, 2011

Kyodai jishin wa naze okiru 巨大地震はなぜ起きる (Pourquoi les séismes géants surviennent-ils?), Tokyo, Kadensha 花伝社.

YAMAMURA Junji 山村順次, 2008 Shin Nihon chiri : shizen kankyō to chiiki hen.yō 新·日本地理: 自然環境亡地域変容 (Nouvelle géographie du Japon : milieux naturels et mutations régionales), Tokyo, Hara shobō 原書房. 
YOSHIMURA Akira 吉村昭, 2011

[10 éd.]

Sanriku kaigan ōtsunami 三陸海岸大津波

(Les tsunamis majeurs sur les côtes du

Sanriku), Tokyo, Bungei shunjū

文藝春秋.

\section{Entretiens cités}

Enquête de terrain du 4 au 10 juillet 2011 (Fukushima-Miyagi-Iwate-Aomori) et du 8 au 11 août 2011 (Iwate-Miyagi)

KATAISHI Keisuke 片石圭介

Responsable de la section de la pêche, Bureau de l'agriculture et de la pêche, ville d'Ōfunato, département d'Iwate, entretien le 9 août 2011.

KOMACHI Takamiki 小町高幹

Responsable de section en charge de la coordination des opérations, Bureau des affaires portuaires, département du développement du front de mer, Gouvernement métropolitain de Tokyo, entretien le 22 juillet 2011.

\section{SAITŌ Hideki 斎藤英紀}

Responsable de la section d'architecture, Bureau de la construction, ville d'Ishinomaki, département de Miyagi, entretien le 11 août 2011.

SATŌ Ken.ichi 佐藤健一 Ingénieur en génie civil, directeur de la cellule de gestion des crises (dues aux catastrophes naturelles), Bureau des affaires générales, ville de Kesennuma, département de Miyagi, entretien le 9 août 2011.

\section{YAMAGUCHI Hiromasa 山口浩雅}

Bureau pour la reconstruction après la catastrophe (du 11 mars 2011), ville d'Ōfunato, département d'Iwate, entretien le 9 août 2011. 